\title{
6. \\ Bemerkung über die Auflösung der biquadratischen Gleichungen.
}

(Von Herrn Dr. S. Aronhold zu Berlin.)

Bezeichnet man die gegebene Gleichung durch
\[ a x^{4}+4 b x^{3}+6 c x^{2}+4 d x+e=0 \]

und berechnet die Determinanten

$$
\Delta=\left|\begin{array}{ccc}
a, & b & c+2 \lambda \\
b, & c-\lambda, & d \\
c+2 \lambda, & d, & e
\end{array}\right|, \quad \frac{\partial A}{\partial e}=\left|\begin{array}{cc}
a, & b \\
b, & c-\lambda
\end{array}\right|
$$

so ist

$$
\Delta=0
$$

eine cubische Gleichung von der Form

$$
\Delta=-4 \lambda^{3}+\boldsymbol{R}+\boldsymbol{Q}=0,
$$

welche, wegen des fehlenden zweiten Gliedes, direct durch die Cardanische Regel aufgelöset werden kann. Sind nun $\left(\frac{\partial \Delta}{\partial e}\right)_{1},\left(\frac{\partial \Delta}{\partial e}\right)_{2},\left(\frac{\partial \Delta}{\partial e}\right)_{3}$ die Werthe der andern Determinante, welche den 3 Wurzeln dieser Gleichung entsprechen, so ist

$$
x=\frac{1}{a}\left\{-b \pm \sqrt{ }\left(\frac{\partial \Delta}{\partial e}\right)_{1} \pm \sqrt{ }\left(\frac{\partial \Delta}{\partial e}\right)_{2} \pm \sqrt{ }\left(\frac{\partial \Delta}{\partial e}\right)_{3}\right\}
$$

wo die zusammengehörigen Zeichen so zu nehmen sind, dafs ihr Product positiv ist.

Noch allgemeiner hat man, für beliebige Werthe von $\xi$ und $\eta$ :

$$
\begin{gathered}
\frac{(a x+b) \xi^{3}+3(b x+c) \xi^{2} \eta+3(c x+d) \xi \eta^{2}+(d x+e) \eta^{3}}{\xi-x \eta} \\
\pm \sqrt{ \pm}\left[\left(\frac{\partial \Delta}{\partial e}\right)_{1} \xi^{4}-\left(\frac{\partial \Delta}{\partial d}\right)_{1} \xi^{3} \eta+\left(\frac{\partial \Delta}{\partial c}\right)_{1} \xi^{2} \eta^{2}-\left(\frac{\partial \Delta}{\partial b}\right)_{1} \xi \eta^{3}+\left(\frac{\partial \Delta}{\partial a}\right)_{1} \eta^{4}\right] \\
\pm \sqrt{ }\left[\left(\frac{\partial \Delta}{\partial e}\right)_{2} \xi^{4}-\left(\frac{\partial \Delta}{\partial d}\right)_{2} \xi^{3} \eta+\left(\frac{\partial \Delta}{\partial c}\right)_{2} \xi^{2} \eta^{2}-\left(\frac{\partial \Delta}{\partial b}\right)_{2} \xi \eta^{3}+\left(\frac{\partial \Delta}{\partial a}\right)_{2} \eta^{4}\right] \\
\pm \sqrt{ }\left[\left(\frac{\partial \Delta}{\partial e}\right)_{3} \xi^{4}-\left(\frac{\partial \Delta}{\partial d}\right)_{3} \xi^{3} \eta+\left(\frac{\partial \Delta}{\partial c}\right)_{3} \xi^{2} \eta^{2}-\left(\frac{\partial \Delta}{\partial b}\right)_{3} \xi \eta^{3}+\left(\frac{\partial \Delta}{\partial a}\right)_{3} \eta^{4}\right]
\end{gathered}
$$


woraus der obige Werth von $x$ folgt, wenn man $\xi=1, \eta=0$ setzt. Für $\xi=0, \eta=1$ erhält man den Werth von $\frac{1}{x}$.

Die Determinanten sind hier übrigens immer so zu bilden, dafs das aus der ersten Diagonale entstehende Glied negativ genommen wird.

Berlin, im Juni 1855. 

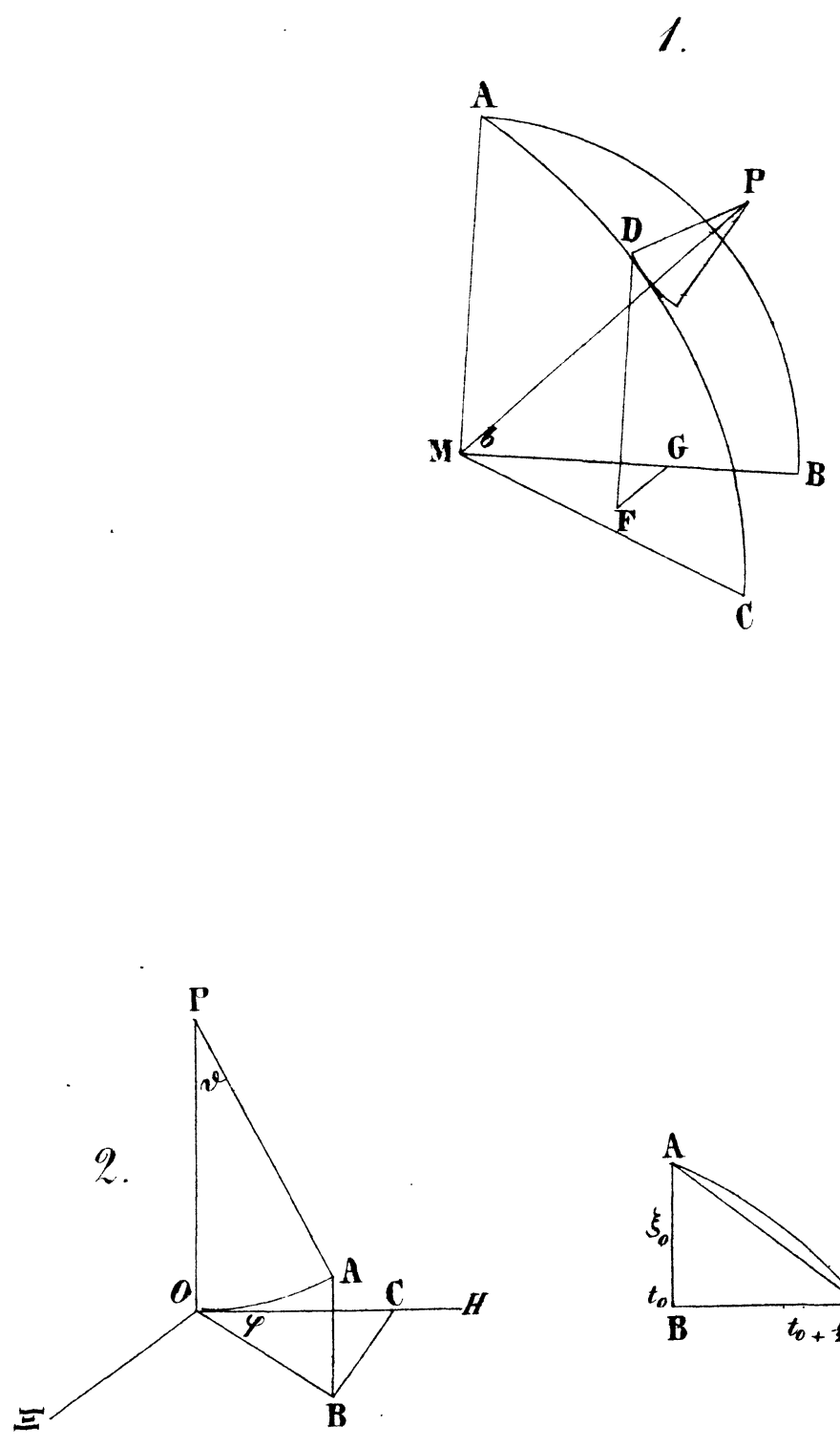

3.

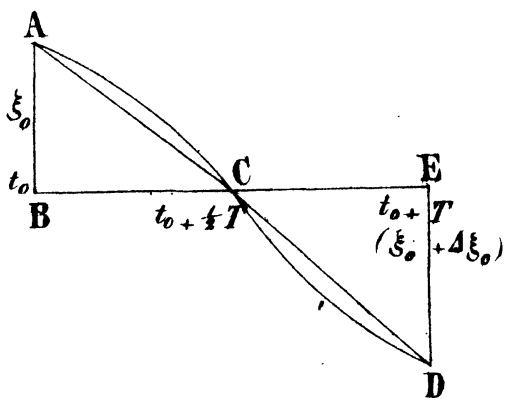




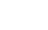

